\title{
Protocols for Harmonized Quantification and Noise Reduction in Low-Dose Oncologic ${ }^{18}$ F-FDG PET/CT Imaging
}

\author{
Marcos A.D. Machado ${ }^{1,2}$, Vinícius O. Menezes ${ }^{1,3}$, Mauro Namías ${ }^{4}$, Naiara S. Vieira ${ }^{1}$, Cleiton C. Queiroz ${ }^{1,5}$, \\ Roberta Matheoud $^{6}$, Adam M. Alessio ${ }^{7}$, and Mércia L. Oliveira ${ }^{8}$ \\ ${ }^{I}$ Nuclear Medicine Department, São Rafael Hospital, Salvador, Brazil; ${ }^{2}$ Hospital das Clínicas da Universidade Federal de \\ Bahia/Ebserh, Salvador, Brazil; ${ }^{3}$ Hospital das Clínicas da Universidade Federal de Pernambuco/Ebserh, Recife, Brazil; ${ }^{4}$ Fundación \\ Centro Diagnóstico Nuclear, Buenos Aires, Argentina; ${ }^{5}$ Hospital Universitario Professor Alberto Antunes/Ebserh, Maceió, \\ Brazil; ${ }^{6}$ Department of Medical Physics, Azienda Ospedaliera Maggiore della Carità, Novara, Italy; ${ }^{7}$ Department of Radiology, \\ University of Washington, Seattle, Washington; and ${ }^{8}$ Centro Regional de Ciências Nucleares (CRCN-NE)/CNEN, Recife, Brazil
}

Oncologic ${ }^{18} \mathrm{~F}-\mathrm{FDG} \mathrm{PET} / \mathrm{CT}$ acquisition and reconstruction protocols need to be optimized for both quantitative and detection tasks. To date, most studies have focused on either quantification or noise, leading to quantitative harmonization guidelines or appropriate noise levels. We developed and evaluated protocols that provide harmonized quantitation with optimal amounts of noise as a function of acquisition parameters and body mass. Methods: Multiple image acquisitions $(n=17)$ of the International Electrotechnical Commission/National Electrical Manufacturers Association PET image-quality phantom were performed with variable counting statistics. Phantom images were reconstructed with 3-dimensional ordered-subset expectation maximization (OSEM3D) and point-spread function (PSF) for harmonized quantification of the contrast recovery coefficient of the maximum pixel value $\left(C R C_{\text {max }}\right)$. The lowest counting statistics that resulted in compliance with European Association of Nuclear Medicine recommendations for $C R C_{\max }$ and $C R C_{\text {max }}$ variability were used as optimization metrics. Image noise in the liver of 48 typical oncologic ${ }^{18} \mathrm{~F}$-FDG PET/CT studies was analyzed with OSEM3D and PSF harmonized reconstructions. We also evaluated 164 additional ${ }^{18}$ F-FDG PET/CT reconstructed list-mode images to derive analytic expressions that predict image quality and noise variability. Phantom-to-subject translational analysis was used to derive optimized acquisition and reconstruction protocols. Results: For harmonized quantitation levels, PSF reconstructions yielded decreased noise and lower $C R C_{\max }$ variability than regular OSEM3D reconstructions, suggesting they could enable a decreased activity regimen for matched performance. Conclusion: PSF reconstruction with a 7-mm postprocessing filter can provide harmonized quantification performance and acceptable image noise levels with injected activity, duration, and mass settings using a $260 \mathrm{MBq} \cdot \mathrm{s} / \mathrm{kg}$ acquisition parameter at scan time. Similarly, OSEM3D with a 5-mm postprocessing filter can provide similar performance with $401 \mathrm{MBq} \cdot \mathrm{s} / \mathrm{kg}$.

Key Words: PET/CT protocols; optimization; quantification; biomarker; EARL accreditation

\footnotetext{
Received Apr. 25, 2018; revision accepted Jul. 26, 2018.

For correspondence or reprints contact: Marcos Machado, Nuclear Medicine Department, Hospital São Rafael, Avenida São Rafael, 2152, São Marcos, Salvador, BA, 41253-190, Brazil.

E-mail: machado@radtec.com.br

Published online Aug. 3, 2018.

COPYRIGHT (c) 2019 by the Society of Nuclear Medicine and Molecular Imaging.
}

J Nucl Med Technol 2019; 47:47-54

DOI: 10.2967/jnmt.118.213405

$\mathbf{P}$ ET and CT hybrid imaging using ${ }^{18} \mathrm{~F}-\mathrm{FDG}$ is an important tool in the management of oncologic patients for clinical diagnosis, staging, prognosis, and treatment response assessment (1-3). Optimal PET/CT imaging should result in consistent diagnostic image quality and quantification with minimal costs and radiation exposure. The use of PET as a quantitative imaging biomarker requires standardization and harmonization of imaging procedures to obtain reproducible quantitative metrics. PET/CT acquisition and reconstruction protocols optimized to meet international quantitative standards might not necessarily be optimized for lesion detection tasks $(4,5)$. The introduction of point-spread function (PSF) modeling in reconstruction algorithms has been shown to increase performance in detecting small and low-intensity lesions (6-9), but SUV quantification of small lesions has lower reproducibility because of increased ensemble noise when low gaussian filters (optimized for detection) are used $(10,11)$.

During the last few years, several facilities have adopted the European Association of Nuclear Medicine (EANM) guidelines through the EANM Research Ltd. (EARL) ${ }^{18} \mathrm{~F}-$ FDG PET/CT accreditation program (12) as a standard for PET SUV quantification (12-14). Despite the wide literature on optimization of PET protocols for lesion detectability $(6-9,15)$ or the impact of noise on quantitation $(16-19)$, there is still limited work optimizing low-dose PET as a quantitative biomarker using guidelines such as the EANM criteria $(20,21)$. Advances in PET image reconstruction algorithms that incorporate PSF modeling have reduced noise and increased resolution, potentially allowing for reduced injected activity and thus lower radiation exposure to patients $(15,22-25)$, opening new scenarios for cancer screening with PET $(15,26,27)$.

PET imaging performance is dependent on the scanner model (6-9), acquisition parameters (administered activity 
and acquisition duration), image reconstruction, and patient habitus (22-25). We have previously suggested acquisition parameter optimization for a particular scanner based on a quadratic or power dose scheme to maintain image noise at a consistent level throughout the population (21,23-25). Image noise is indeed a source of variance in the SUV quantification of tumors and ideally should be maintained at a constant level over the patient population to ensure consistent quantification variability (16-19). In general, the reconstruction settings define whether the contrast recovery coefficients are within the harmonization criteria (20). Lasnon et al. demonstrated that PSF reconstruction can result in images in quantitative compliance with the EANM guidelines using additional gaussian filtering (28). However, this approach requires that the detection and quantitative tasks be performed on 2 different image volumes. Thus, a commercial solution (EQ.PET; Siemens Healthcare) allowing all-in-one diagnostic and quantitative images was validated by Quak et al., who found that PSF modeling could be used to provide higher detectability and the EANM quantitative recommendations at the same time $(29,30)$. Our initiative is also in line with the increasing interest in task-specific protocols, which can reduce costs and increase diagnostic accuracy (26).

The present study aims at developing optimized imaging protocols providing recommendations on acquisition and reconstruction settings to obtain lower injected activities and harmonized quantification according to the EANM guidelines. The EARL procedure specifies that image noise should not exceed $15 \%$ and that recovery coefficients should remain unbiased (21). To account for differences in the noise behavior between standard 3-dimensional ordered-subset expectation maximization (OSEM3D) and PSF reconstructions, we extended the concept of harmonization by defining optimal quantification variability levels, which are affected by image noise. Therefore, this work aimed to characterize the noise properties of PSF reconstructions, verify the consistency of a newly defined harmonization bias index $(H B I)$ across acquisition parameters for both OSEM3D and PSF reconstructions, and define reconstruction-specific formulas for optimal acquisition parameters that satisfy $H B I$, noise levels, and quantification variability.

This work provides a feasible methodology that might be applied in PET centers to decrease the injected activity while achieving consistent image quality and quantification when using PSF reconstructions.

\section{MATERIALS AND METHODS}

\section{Scanner}

PET/CT imaging was performed on a Biograph TruePoint TrueV PET scanner (Siemens) combined with a 16-slice CT scanner (Emotion 16; Siemens). PET images were corrected for random coincidences, normalization, dead-time losses, scatter, and attenuation. The attenuation map was obtained by a spiral CT scan $(100 \mathrm{kVp}$, automatic tube-current modulation) on a $168 \times$ 168 matrix $(4.07 \times 4.07 \times 3 \mathrm{~mm}$ voxels $)$ and a standard soft- tissue reconstruction kernel (Siemens B30s) for both phantom and patients. All images were acquired within 3 mo from the cross-calibration between PET scanner and dose calibrator.

\section{Phantom Study}

We used an International Electrotechnical Commission/National Electrical Manufacturers Association body phantom with 6 spheres (internal diameters of $10,13,17,22,28$, and $37 \mathrm{~mm}$ ) filled with an ${ }^{18}$ F-FDG stock solution of $45 \mathrm{MBq}$ diluted in $1,000 \mathrm{~mL}$ and the phantom compartment filled with $42 \mathrm{MBq}$ of ${ }^{18} \mathrm{~F}$-FDG to yield a sphere-to-background concentration ratio of 10:1, according to the EANM guideline (20). Hereafter, we define the phantom activity as the ${ }^{18} \mathrm{~F}$-FDG activity in the background. The contrast recovery coefficient of the maximum pixel value $\left(C R C_{\max }\right)$ is defined as the ratio between the measured activity concentration in the maximum pixel and the actual activity concentration in the sphere. Thus, $C R C_{\text {max }}$ represents the fraction of $\mathrm{SUV}_{\max }$ recovery of a given lesion size in patient scans.

Four different cumulated counting levels (groups A-D) were acquired with multiple acquisitions to evaluate the variance of $C R C_{\text {max }}$. Sets of PET/CT images were sequentially acquired at 2 bed positions. The phantom activity and the image duration were adjusted to reproduce equivalent counting within groups as follows: group A, 1,272 MBq.s/kg, high count (2 frames at 26.7 and 23.4 $\mathrm{MBq}$ for 449 and $510 \mathrm{~s}$ each); group $\mathrm{B}, 416 \mathrm{MBq} \cdot \mathrm{s} / \mathrm{kg}$, clinical standard (5 frames at 20.0, 19.5, 18.3, and 16.5 MBq for 190, 202, 220, 225, and $235 \mathrm{~s}$ each); group C, $216 \mathrm{MBq} \cdot \mathrm{s} / \mathrm{kg}$ (5 frames at $12.6,12.2,11.7,11.2$, and $10.7 \mathrm{MBq}$ for $160,165,170,180$, and $200 \mathrm{~s}$ each); and group D, $81 \mathrm{MBq} \cdot \mathrm{s} / \mathrm{kg}$ (5 frames at 14.3, 14.1, 13.9, 13.7, and 13.5 MBq for 76, 80, 81, 83, and $86 \mathrm{~s}$ each).

Acquisition parameters in the phantom-defined as the product between the phantom activity at acquisition time (in megabecquerels) and the acquisition duration per bed position (in seconds) were normalized by $9.4 \mathrm{~kg}$, which is the phantom weight in the background.

\section{Reconstruction}

Images were reconstructed with the following algorithms: OSEM3D (with 3 iterations, 21 subsets, and a 5-mm gaussian filter [EARL-harmonized]); PSF7 (PSF ordinary Poisson with 3 iterations, 21 subsets, and a 7-mm gaussian filter [EARLharmonized]); and PSF2 (PSF ordinary Poisson with 2 iterations, 21 subsets, and a 2-mm gaussian filter). In addition, the PSF2 filter had additional filtering applied with the EQ.PET package (PSF2-EQ*, where the asterisk denotes the amplitude of gaussian filtering).

The OSEM3D reconstruction is our current clinical standard, and the PSF2 is used for detection optimization $(9,24)$. All gaussian filters were 3-dimensional isotropic.

\section{Phantom Image Analysis}

The $C R C_{\max }$ of the hot spheres was calculated using an automatic routine provided by the EANM on request (31), except for the PSF2 images, which were analyzed with EQ.PET (Siemens) by applying varying postprocessing filters $(6,6.5$, and $7 \mathrm{~mm})$ to determine the configuration that complies with the EANM quantification standards.

The protocol was considered harmonized according to EARL standards if the mean $C R C_{\max }$ was within the EARL limits. We defined an overall $H B I$ to measure, for each acquisition and reconstruction protocol, the deviation of the $C R C_{\max }$ of each sphere from the corresponding EARL limits: 


$$
H B I=\frac{1}{6} \times \sum_{i=1}^{6} \frac{\left|C_{i}-L_{i}\right|+\left|C_{i}-U_{i}\right|}{K_{i}}
$$

where $i=1-6$ indicates each phantom sphere, $C_{i}$ is the average of $C R C_{\text {max }}$ for sphere " $i$ " obtained through repeated acquisitions, $U_{i}$ and $L_{i}$ are the upper and lower EARL limits for sphere " $i$," and $K_{i}$ is a normalization index calculated as the difference between $U_{i}$ and $L_{i}$ limits for sphere " $i$." According to this definition, one expects $H B I$ to be 1.00 if the protocol is EARL-compliant, and the higher the $H B I$ is, the greater is the bias from EARL limits.

The ratio between the standard deviation and the average of the $C R C_{\max }$ values of sphere " $\mathrm{i}$ " across realizations were calculated and averaged among all 6 spheres, for each acquisition group, to estimate the quantification variability $\left(Q_{v a r}\right)$ of a protocol as follows:

$$
Q_{v a r}=\frac{1}{6} \times \sum_{i=1}^{6}\left(\frac{\sigma_{i}}{C_{i}}\right)
$$

where $\sigma_{i}$ is the SD of $C R C_{\max }$ across realizations for sphere $i$.

Additionally, according to PERCIST criteria 1.0 (32), we defined a spheric volume of interest of $3-\mathrm{cm}$ diameter in the background (Fig. 1) to compute the background noise metric $C V$, defined as

$$
C V=\frac{\sigma_{b}}{\mu_{b}}
$$

where $\sigma_{b}$ and $\mu_{b}$ are the SD and the mean pixel value in the volume of interest, respectively.

\section{Patient Imaging}

Conventional ${ }^{18} \mathrm{~F}$-FDG PET patient examinations for wholebody oncologic workup were randomly selected and excluded on the basis of the following criteria: pregnancy or nursing, motor difficulties, liver metastases, hyperglycemia at the time of tracer administration, or a delay exceeding $90 \mathrm{~min}$ between ${ }^{18} \mathrm{~F}$-FDG injection and image acquisition. The study was approved by the Monte Tabor-Hospital São Rafael ethical board, and all subjects gave written informed consent. The images were acquired according to the clinical protocol of Hospital São Rafael for tumor PET imaging. Subjects fasted for $6 \mathrm{~h}$ before the ${ }^{18} \mathrm{~F}-\mathrm{FDG}$ injection.

\section{Prospective Patient Imaging (Step 1)}

In total, $48{ }^{18} \mathrm{~F}-\mathrm{FDG}$ PET/CT whole-body adult studies (mean body mass, $69 \pm 15 \mathrm{~kg}$; range, 36-102 kg) were gathered over a 2 -mo period and prospectively evaluated. Images were acquired from the mid thigh to the vertex of the skull of subjects who lay supine with the arms positioned above the head. PET scans started $67 \pm 10 \mathrm{~min}$ (range, $51-90 \mathrm{~min}$ ) after injection of $3.1 \pm 0.7 \mathrm{MBq} / \mathrm{kg}$ (range, 1.4-5.8 MBq/kg), and the acquisition duration was adjusted according to the methodology of reference 24 for a given patient

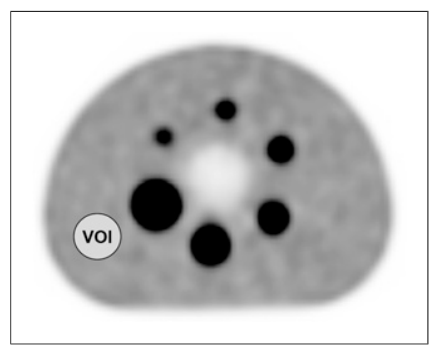

FIGURE 1. Positioning of 3$\mathrm{cm}$-diameter volume of interest (VOI) for CV calculation. weight and injected activity, with the mean duration being $201 \pm$ $43 \mathrm{~s}$ (range, 111-300 s).

\section{Retrospective List-Mode Imaging (Step 2)}

This step aimed at characterizing the noise levels of PSF7 as a function of the acquisition parameters. We extracted 41 list-mode images of the liver region from a database of our previous work (24). The subjects weighed $70 \pm 16 \mathrm{~kg}$ (range, 45-120 kg), and the injected activity was $3.5 \pm 0.6 \mathrm{MBq} / \mathrm{kg}$ (range, $2.3-4.6 \mathrm{MBq} / \mathrm{kg}$ ). All 41 list-mode images were acquired for $360 \mathrm{~s}$ and reconstructed at incremental 1-min intervals up to $4 \mathrm{~min}$ (4 images per patient) with PSF7 reconstruction, resulting in 164 images.

\section{Patient Image Analysis}

All images were analyzed with Syngo.VIA, version VA30 (Siemens Healthcare). The $C V$ was measured in the liver of healthy subjects using the same volume-of-interest size as used for the phantom experiments.

For step 1, OSEM3D and PSF7 were used to analyze the differences in $C V$. Because we aimed at comparing the noise characteristics between both harmonized reconstructions (similar spatial resolution), the PSF2 algorithm was not evaluated and can be assessed elsewhere (24).

For step 2, $C V$ was analyzed through a first-order approximation for the $C V$ from the total collected counts, as previously published (24):

$$
C V=a \times(C D)^{b}, \quad \text { Eq. } 4
$$

where $a$ and $b$ are the regression coefficients, $C D$ is the acquisition parameter in $\mathrm{MBq} \cdot \mathrm{s} / \mathrm{kg}$ at imaging time (injected activity corrected for physical decay), and $C V$ was obtained according to Equation 3. Data available from step 1 were used to cross validate the analytic curve obtained in step 2. Additionally, the $C V$ values of step 2 were divided into 3 groups of $C D(<200 \mathrm{MBq} \cdot \mathrm{s} / \mathrm{kg}[n=$ 45], 200-300 MBq.s/kg [ $n=32]$, and 300-400 MBq.s $/ \mathrm{kg}[n=$ $33]$ ), and the dispersion of $C V$ within groups was verified by computing the $C V$ variance $\left(\sigma^{2}{ }_{\mathrm{cv}}\right)$ :

$$
\sigma_{c v}^{2}=\frac{\sum(C V-\overline{C V})^{2}}{N-1}
$$

where $\overline{C V}$ is the averaged $C V$ and $N$ is the number of $C V$ measurements within a group.

In clinical imaging, $C V$ is a function of injected activity, acquisition time, reconstruction, processing, and patient-dependent parameters (e.g., body mass). For a particular $C V$ and scanner settings, the expected activity and acquisition duration to achieve matched noise levels as a function of object mass is as follows (23):

$$
A \cdot t=\alpha \times m^{\beta},
$$

Eq. 6

where $A$ is the injected activity at scan time, $t$ is the acquisition duration, $\alpha$ and $\beta$ are scanner-specific coefficients, and $m$ is the body mass. Previous published data for PSF2 and OSEM3D suggested acquisition parameters that give a $C V$ of $12 \%$ as optimal (24). These acquisition parameters were arranged in Equation 6 to benchmark current recommendations, and the results from steps 1 and 2 were used to define $A \cdot t$ for PSF7.

Figure 2 illustrates the design of the study.

\section{Statistical Analysis}

The residuals of regression were tested for normality using the Shapiro-Wilk test to validate the curve fit of $C V$ in the liver. An 


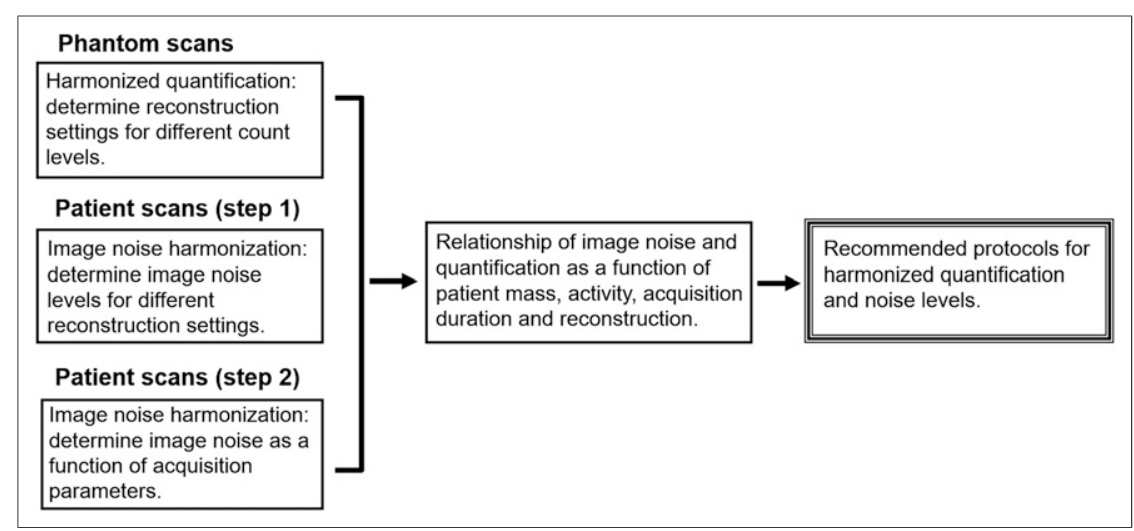

FIGURE 2. Flow chart to achieve low-dose and task-specific protocols. struction setting, the image noise depends on the activity concentration, acquisition duration, scatter, and attenuation conditions, which are similar between the phantom and a $75-\mathrm{kg}$ standard patient because of their similar transaxial section. At scan time, the injected activity concentration in patients was similar to that in the phantom $(\sim 2 \mathrm{MBq} / \mathrm{kg})$. However, because the SUV is the ratio of local concentration to injected activity concentration, a higher SUV indicates a higher concentration relative to the injected activity. Therefore, the liver had 2-3 times more ${ }^{18}$ F-FDG activity (SUV,
$F$ test was performed to check for differences between the $\sigma^{2}{ }_{\mathrm{cv}}$ for the $200-300 \mathrm{MBq} \cdot \mathrm{s} / \mathrm{kg}$ group and the following or previous groups. Graphs and statistical analysis were performed with OriginPro, version 9.0.0 (OriginLab Corp.), and GraphPad Prism, version 5.01. Where indicated, statistics of $C V$ were computed following the limits for $95 \%$ confidence interval, and a $P$ value of less than 0.05 was considered statistically significant.

\section{RESULTS}

\section{Phantom Studies}

Figure 3 shows the $C R C_{\text {max }}$ curves of each protocol. The EQ.PET filter that harmonized PSF2 with minimal bias was $6.5 \mathrm{~mm}$ (PSF2-EQ6.5), as shown by the HBIs reported in Table 1 and Figure 4, with the HBI for this reconstruction setting being the lowest among the tested EQ.PET filters.

Noise levels in uniform regions are commonly used as an indicator of quantification variability, and uniform region noise having a $C V$ of $15 \%$ has been suggested as a limiting noise level (21). Figure 4 demonstrates that the $Q_{v a r}$ for the PSF reconstructions was lower than that for OSEM3D for all groups of acquisition parameters (e.g., for group $\mathrm{C}$, the noise with PSF is lower than OSEM3D), leading to less reconstruction-dependent variability. Interpolation of points for OSEM3D in Figure $4 \mathrm{~A}$ suggests that a $Q_{v a r}$ of $4 \%$ would be a more reliable limit regardless of the reconstruction method. For quantifications with EQ.PET, it is important to stress that EQ.PET performs quantifications only on the spheres (lesions), and the background noise for PSF2 is presented as with only 2-mm smoothing in Figure 4.

\section{Patient Studies}

Step 1. The injected activity and acquisition duration resulted in a decay-corrected acquisition parameter of $397 \pm$ $68 \mathrm{MBq} \cdot \mathrm{s} / \mathrm{kg}$ (range, 221-612 MBq·s $/ \mathrm{kg}$ ). The evaluation of background noise in the liver for step 1 resulted in a $C V$ of $11.7 \% \pm 3.6 \%$ and $6.7 \% \pm 2.4 \%$ (confidence interval $=$ $95 \%$ ) for OSEM3D and PSF7, respectively (Fig. 5). Thus, noise in the liver is 1.75 times higher for OSEM3D than for PSF7 $(P<0.0001)$.

Step 2. The plots of liver noise from step 2 (Fig. 6) show trends similar to the phantom studies. For a given recon-
2-3) than the phantom background (SUV, 1). For the liver region, the presence of activity in adjacent volumes penalizes the noise-equivalent count rate and negatively affects image noise. Noise-equivalent count rate and image noise

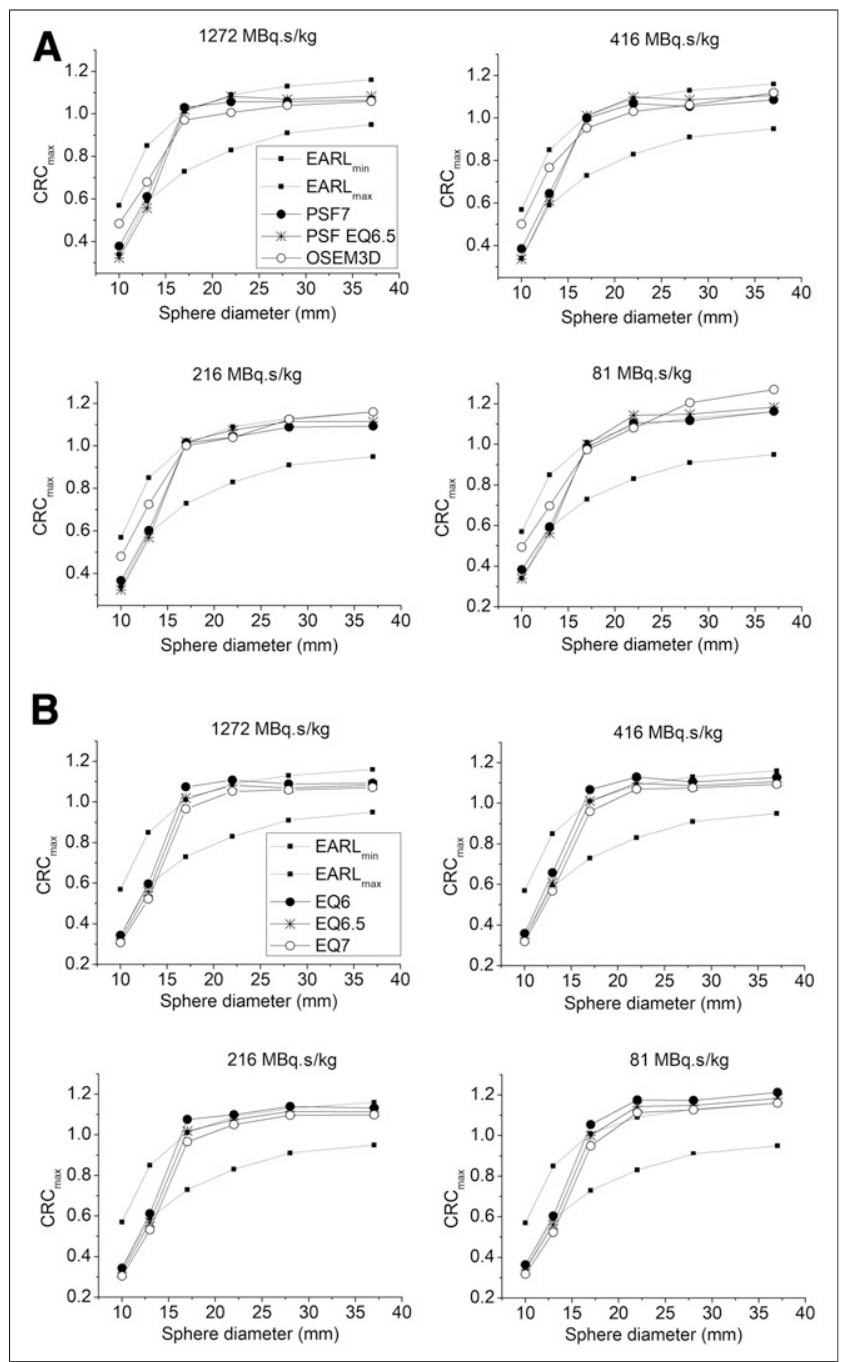

FIGURE 3. $C R C_{\max }$ for OSEM3D, PSF7, and PSF2-EQ* protocols. 
TABLE 1

HBls for Groups of Acquisition Parameters and Different Reconstruction Settings

\begin{tabular}{cccccc}
\hline Acquisition parameter & OSEM3D & PSF7 & PSF2-EQ6 & PSF2-EQ6.5 & PSF2-EQ7 \\
\hline Group A: $1,272 \mathrm{MBq} \cdot \mathrm{s} / \mathrm{kg}$ & & 1.02 & 1.13 & 1.08 & 1.13 \\
Group B: $416 \mathrm{MBq} \cdot \mathrm{kg}$ & 1.00 & 1.00 & 1.17 & 1.02 & 1.06 \\
Group C: $216 \mathrm{MBq} \cdot \mathrm{s} / \mathrm{kg}$ & 1.00 & 1.01 & 1.20 & 1.06 & 1.18 \\
Group D: $81 \mathrm{MBq} \cdot \mathrm{s} / \mathrm{kg}$ & 1.29 & 1.02 & 1.40 & 1.15 \\
\hline
\end{tabular}

have different relations according to reconstruction, with images reconstructed using PSF modeling being less affected by noise-equivalent count rate degradation $(22,24)$. Areas of inhomogeneous ${ }^{18} \mathrm{~F}-\mathrm{FDG}$ uptake and anatomic noise in the liver are another source of increased noise in the clinical situation. However, the higher ${ }^{18} \mathrm{~F}-\mathrm{FDG}$ activity in the liver dominates over these factors in patients in comparison to the phantom.

\section{Noise Standardization and Protocol Validation}

By comparing step $1(397 \pm 68 \mathrm{MBq} \cdot \mathrm{s} / \mathrm{kg}$; group B; $C V$, $6.7 \% \pm 2.4 \%$; confidence interval, $95 \%$ ) and step 2 for the same range of $C D(388 \pm 36 \mathrm{MBq} \cdot \mathrm{s} / \mathrm{kg} ; n=39 ;$ body mass, $73 \pm 18 ; C V, 7.0 \% \pm 2.4 \%$, confidence interval, $95 \%$ ), one can conclude that the 2 groups have clinically equivalent noise performance. A 2-sided $t$ test was performed to rule out the null hypothesis that the difference in these 2 groups is greater than $0.7 \%$ (indifference zone). As such, we find that the mean difference in the groups was $0.3 \% \pm$ $0.3 \%$, deemed to be equivalent by this definition $(P<0.05)$.

The images of subjects weighing more than $100 \mathrm{~kg}$ (mean, $113 \pm 12 \mathrm{~kg} ; n=3 ; C D, 378 \pm 57 \mathrm{MBq} \cdot \mathrm{s} / \mathrm{kg}$ ) were selected and found a $C V$ of $7.3 \% \pm 1.9 \%$ (confidence interval, $95 \% ; k=4.3$ ) as predicted by the analytic model where $C V$ is $7.2 \%$ (Fig. 6). These findings support the hypothesis of a linear model for PSF7. Hence, coefficient $\beta$ in Equation 6 may be adjusted to $\beta=1$, and $C D$ in PSF7 may be linearly adjusted according to patient body mass.

Analysis of $\sigma^{2}$ cv as a function of $C D$ showed a higher $\sigma^{2}{ }_{\text {cv }}$ when $C D$ was less than $200 \mathrm{MBq} \cdot \mathrm{s} / \mathrm{kg}$, where $\sigma^{2}{ }_{\mathrm{cv}}$ was $3.6 \times 10^{-4}, 1.4 \times 10^{-4}$, and $1.6 \times 10^{-4}$ for a $C D$ of less than 200, 200-300, and 300-400 MBq·s/kg, respectively, and the mean value of the $C D$ groups was $136.6,256.2$, and
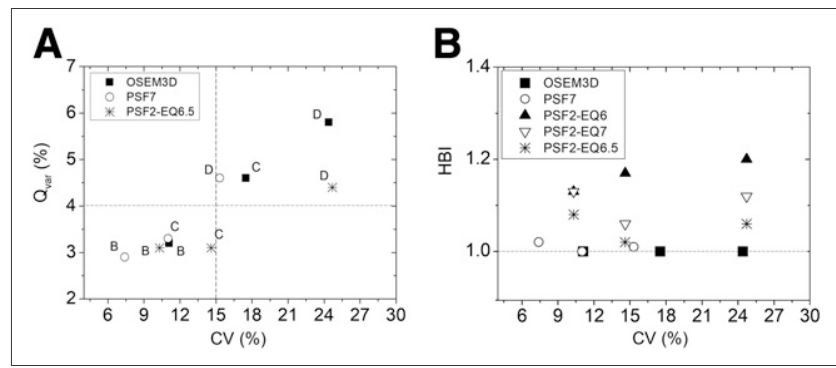

FIGURE 4. Quantification variability $(\mathrm{A})$ and $H B I(\mathrm{~B})$ vs. image noise. Plots represent $C V$ generated from acquisitions of groups B, C, and D. Dashed lines represent limits of acceptability for $Q_{v a r}$ (A) and $H B I(B)$, where a $C V$ of $15 \%$ is the EARL reference.
355.4 MBq·s $/ \mathrm{kg}$, respectively. The $F$ test demonstrated that $\sigma_{c v}^{2}$ was statistically different between less than 200 and 200-300 MBq.s/kg $(P=0.007)$. However, no significant difference in $\sigma_{c v}^{2}$ was observed when 200-300 and 300$400 \mathrm{MBq} \cdot \mathrm{s} / \mathrm{kg}$ were compared $(P=0.69)$. From this analysis, we can state that a $C D$ higher than $260 \mathrm{MBq} \cdot \mathrm{s} / \mathrm{kg}$ will provide a $C V$ with acceptable variability for PSF7.

\section{Optimal ${ }^{18}$ F-FDG Dose Schemes}

Both PSF configurations yielded lower doses than those commonly used in PET studies (Table 2). For optimal PSF2 imaging, $326 \mathrm{MBq} \cdot \mathrm{s} / \mathrm{kg}(\beta=1.48)$ provides a $C V$ of $12 \%$ in the liver, considered the gold standard (24). The optimal $C D$ for PSF7 is $260 \mathrm{MBq} \cdot \mathrm{s} / \mathrm{kg}(\beta=1)$, which will result in a 106,158 , and $211 \mathrm{MBq}$ injected activity for, respectively, a 50, 75 , and $100 \mathrm{~kg}$ body mass. Thus, PSF could guide the design of new low-dose PET protocols in the range if $260-326 \mathrm{MBq} \cdot \mathrm{s} / \mathrm{kg}$ for a $75-\mathrm{kg}$ standard patient and $\beta$ between 1 and 1.5 .

Figure 7 shows the injected activity for each reconstruction protocol. Ideally, lesion detectability should be maximized using PSF with minimum filtering (9) while maintaining SUV harmonization (EQ.PET) and injected activity as low as possible. On the other hand, detectability relies on a tradeoff among injected activity and reconstruction preferences (8). Therefore, PSF7 would provide the lowest injected activity and image quality still within international standards.

\section{DISCUSSION}

This study aimed to derive optimal ${ }^{18} \mathrm{~F}-\mathrm{FDG}$ PET/CT imaging protocols as a function of body mass to achieve quantification consistent with the EARL criteria while keeping the image noise standardized. The use of harmonized metrics may help with personalized medicine since these enable more reliable assessment of tumor characteristics. Advances in hardware and image reconstruction in PET technology can allow for decreased ${ }^{18} \mathrm{~F}-\mathrm{FDG}$ injected activity, but the exact influence of PSF for ${ }^{18} \mathrm{~F}-\mathrm{FDG}$ dose optimization and harmonized quantification is still unclear.

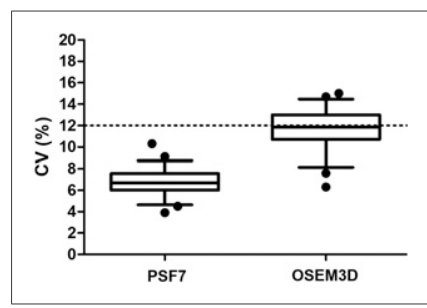

FIGURE 5. Comparison of $C V$ between PSF7 and OSEM3D. Dashed line indicates OSEM3D and PSF2 noise obtained from formulations in reference 24 . 


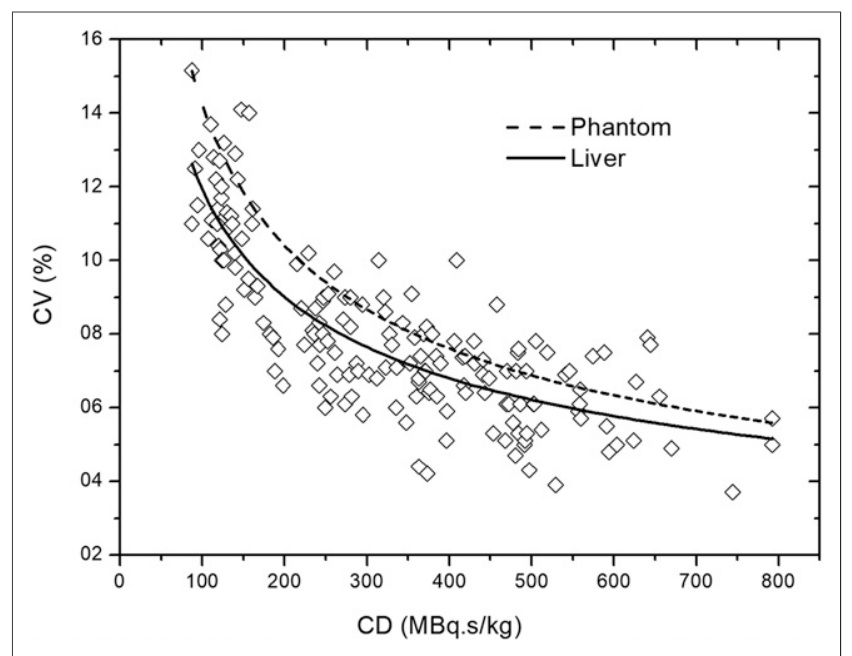

FIGURE 6. Plot of liver noise (CV) vs. acquisition parameter for patient data from step 2 using PSF7. Curves fit to these liver data (solid line) and to phantom data (dashed line) were generated with fitting coefficient of $a=0.77, b=-0.40, R^{2}=$ 0.67 , and $a=1.40, b=-0.49, R^{2}=0.98$ for liver and phantom, respectively.

\section{EARL Harmonization and Patient-Specific Dose Schemes}

The overall compliance rate of EARL applicants is reported as only $63 \%$, because of contrast recovery coefficient inconsistencies (14). Other authors have published data with EARL quantification slightly outside the EARL limits $(14,28,33)$, but they did not state the magnitude of bias. In this work, we defined an $H B I$ to quantify agreement with the EARL standards. It is important to note that the $H B I$ metric has limitations in objectively comparing different bias configurations. For example, multiple spheres could be slightly biased and have the same $H B I$ as a configuration in which one sphere is very biased; also, $H B I$ does not tell the direction of bias (e.g., whether recovery coefficients are too low or too high). However, the proposed metric is useful to address the magnitude of the "slight bias" frequently reported in the literature.

For patient studies, in which patient habitus strongly affects the noise-equivalent count rate curves, higher injected activity will result in loss of data quality, which influences image quality. Therefore, increasing the acquisition duration is more effective than increasing the injected activity to preserve the image quality (25). That is, the $\beta$-value depends on the acquisition duration and the injected activity, and $\beta$ will be smaller if the injected activity is limited to smaller values. In our facility, the injected activity for larger patients $(>90 \mathrm{~kg}$ ) is limited to $3 \mathrm{MBq} / \mathrm{kg}$, and the acquisition duration is adjusted accordingly to maintain the counting statistics.

\section{Reconstruction-Specific Dose Schemes}

We showed that reconstructing the same raw data with PSF2 yielded an acquisition parameter that was more linear as a function of mass than OSEM3D (24). In other words, the PSF2 reconstruction had a lower $\beta$-value in the PSF reconstruction (Eq. 6). Thus, the optimal acquisition parameter for noise standardization should be adjusted according to patient-specific parameters (e.g., body mass) using reconstruction-specific power equations, contrary to the generalized quadratic method $(21,23)$.

In this work, we used 164 datasets ( 4 images per patient) to obtain the noise curve with count levels that mimic a clinical situation. In Figure 6, differences of coefficient $\beta$ between phantom and liver fits obtained with Equation 4 could be attributed to the fact that patient mass may not be sufficient to normalize across very different morphologies. However, the residuals of regression were normally distributed in the Shapiro-Wilk test of normality $(\mathrm{w}=0.359$, $P<0.05)$.

We compared the performance of regular OSEM3D and low-dose PSF configurations regarding EARL compliance, $Q_{v a r}$, and $\sigma_{c v}^{2}$ in the liver. Our results showed that a linear approach is feasible for PSF7 with $260 \mathrm{MBq} \cdot \mathrm{s} / \mathrm{kg}$, which performs similarly to OSEM3D with $400 \mathrm{MBq} \cdot \mathrm{s} / \mathrm{kg}$. However, the small cohort of patients weighing more than 100 $\mathrm{kg}(n=3)$ was a limitation in our study, encouraging its future validation on a larger population.

We demonstrated that strong filtering (PSF2-EQ6.5 and PSF7) improved the SUV variability of PSF over OSEM3D for the same counting level. This consideration is especially important for monitoring of a disease or designing new protocols when low dose is required, such as the recent interest in screening with PET.

\section{Final Considerations}

Clinical SUV measurements are prone to numerous sources of error related to technical, physical, and biologic factors (34-37). Although the EANM guideline recommends following strict technical factors (cross calibration, clocks synchronization, and others), the physical related factors (acquisition and reconstruction parameters) mirrored in the contrast recovery coefficient values are quite wide, and differences between the minimum and maximum

TABLE 2

Optimal Counting Levels at Scan Time and Derived ${ }^{18}$ F-FDG Injected Activity

\begin{tabular}{lllll}
\hline & & \multicolumn{3}{c}{ Body mass } \\
\cline { 3 - 4 } Parameter & Equation & $50 \mathrm{~kg}$ & $75 \mathrm{~kg}$ & $100 \mathrm{~kg}$ \\
\hline PSF7 & $A \cdot t=260 \cdot m$ & $106 \mathrm{MBq}$ & $158 \mathrm{MBq}$ & $211 \mathrm{MBq}$ \\
PSF2 (24) & $A \cdot t=41 \cdot m^{1.48}$ & $111 \mathrm{MBq}$ & $189 \mathrm{MBq}$ & $300 \mathrm{MBq}$ \\
OSEM3D (24) & $A \cdot t=12 \cdot m^{1.82}$ & $126 \mathrm{MBq}$ & $244 \mathrm{MBq}$ & $426 \mathrm{MBq}$
\end{tabular}

$A=$ activity at scan time (MBq); $t=$ acquisition duration (s); $m=$ body mass.

Coefficients $\alpha$ and $\beta$ (Eq. 7) for OSEM3D and PSF2 were determined through regression of data from Table $V$ in reference 24. Injected activity is decay-corrected for 60 min before scan time and typical 3-min acquisition duration per bed position. 


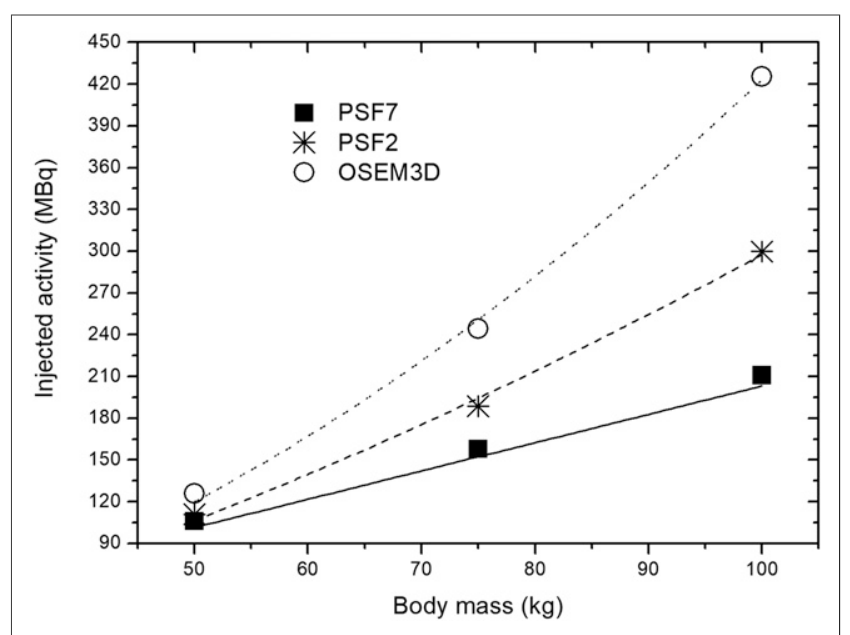

FIGURE 7. Dose scheme illustration. Injected activity is shown for 60-min uptake time and normalized to 3-min duration per bed acquisition.

EARL limits are within $20 \%-45 \%$ from the largest to the smallest spheres (12-14), showing that one must still be cautious when quantifying lesions with different protocols.

In the context of EARL accreditation, it is challenging that a large pool of imaging systems converges to the "same" contrast recovery coefficient curve (14). The implementation of PSF reconstruction with narrow gaussian filtering amplitudes allows for the smaller spheres of the National Electrical Manufacturers Association phantom to reach higher contrast recovery coefficient values, but the Gibbs effects overshoots quantification of the $17-\mathrm{mm}$ sphere (38). This issue was resolved with strong gaussian filtering, and consequently, the contrast recovery coefficients of the 10- and 13-mm spheres were close to the lower contrast recovery coefficient limits. The EQ.PET filter, however, may easily be set up with a narrower filter when quantifying smaller lesions $(<13 \mathrm{~mm})$ in the same reconstructed image.

Of course, the EARL limits are not ideal but are a feasible recommendation for most PET-scanner generations to achieve minimal standards. On the other hand, alternative reconstruction configurations are encouraged to match the contrast recovery coefficient with minimal differences (34).

This work did not directly assess the relationship between acquisition and reconstruction parameters and detectability. Ideally, we would find protocols that achieve a sufficient level of quantification and detectability performance. We fall short of this goal using quantification and background noise as a surrogate for detectability under the assumption that quantification and background noise are reflective of contrast-to-noise ratio, a metric correlated with detectability. Although this shortfall is a limitation, our work represents one of the few studies attempting to find protocols with appropriate quantification and background noise levels.

Acquisition parameter adjustments according to patient habitus and reconstructions are required to maintain standardized image noise throughout the patient population and ensure consistent image quality and quantifications. Our method to achieve low-dose PSF-based protocols will also allow for management to improve the PET facility by scanning patients faster or decreasing the patient dosimetry and radiopharmaceutical costs.

\section{CONCLUSION}

This study provides scanner and patient-specific recommendations for an optimized oncologic ${ }^{18} \mathrm{~F}-\mathrm{FDG}$ PET/CT imaging protocol when PSF modeling reconstruction is applied, with the aim of achieving compliance with the EARL standards. Optimal PET acquisition and reconstruction settings will allow for reduction of scan duration or administered ${ }^{18} \mathrm{~F}-$ FDG activity and consistent assessment of metabolic tumor activity, aligned with demands for personalization of patient assistance.

\section{DISCLOSURE}

No potential conflict of interest relevant to this article was reported.

\section{ACKNOWLEDGMENTS}

We thank the Comissao Nacional de Energia Nuclear (CNEN, Brazil) and the International Atomic Energy Agency (IAEA) for their efforts to develop nuclear medicine at our institutions through a technical cooperation project. We also thank both reviewers for their crucial comments, which improved the quality of our work.

\section{REFERENCES}

1. Ambrosini V, Nicolini S, Caroli P, et al. PET/CT imaging in different types of lung cancer: an overview. Eur J Radiol. 2012;81:988-1001.

2. Weber WA. Use of PET for monitoring cancer therapy and for predicting outcome. J Nucl Med. 2005;46:983-995.

3. de Geus-Oei LF, van der Heijden HF, Corstens FH, Oyen WJG. Predictive and prognostic value of FDG-PET in nonsmall-cell lung cancer: a systematic review. Cancer. 2007;110:1654-1664.

4. Boellaard R. The engagement of FDG PET/CT image quality and harmonized quantification: from competitive to complementary. Eur J Nucl Med Mol Imaging. 2016;43:1-4.

5. Boellaard R. Optimisation and harmonisation: two sides of the same coin? Eur J Nucl Med Mol Imaging. 2013;40:982-984.

6. Andersen FL, Klausen TL, Loft A, Beyer T, Holm S. Clinical evaluation of PET image reconstruction using a spatial resolution model. Eur J Radiol. 2013;82: 862-869.

7. Schaefferkoetter J, Casey M, Townsend D, Fakhri G. Clinical impact of time-offlight and point response modeling in PET reconstructions: a lesion detection study. Phys Med Biol. 2013;58:1465-1478.

8. Adler S, Seidel J, Choyke P, et al. Minimum lesion detectability as a measure of PET system performance. EJNMMI Phys. 2017;4:13.

9. Kadrmas DJ, Casey ME, Conti M, Jakoby BW, Lois C, Townsend DW. Impact of time-of-flight on PET tumor detection. J Nucl Med. 2009;50:1315-1323.

10. Tong S, Alessio AM, Kinahan PE. Noise and signal properties in PSF-based fully 3D PET image reconstruction: an experimental evaluation. Phys Med Biol. 2010; 55:1453-1473.

11. Rahmim A, Qi J, Sossi V. Resolution modeling in PET imaging: theory, practice, benefits, and pitfalls. Med Phys. 2013;40:064301.

12. EANM FDG PET/CT accreditation specifications for SUV recovery coefficients. EANM research Ltd. website. http://earl.eanm.org/cms/website.php?id=/en/projects/ fdg_pet_ct_accreditation/accreditation_specifications.htm. Published January 2017. Accessed December 6, 2018. 
13. Boellaard R, Hristova I, Ettinger S, et al. EARL FDG-PET/CT accreditation program: feasibility, overview and results of first 55 successfully accredited sites [abstract]. J Nucl Med. 2013;54(suppl 2):2052.

14. Kaalep A, Sera T, Oyen W, et al. EANM/EARL FDG-PET/CT accreditation: summary results from the first 200 accredited imaging systems. Eur J Nucl Med Mol Imaging. 2018;45:412-422.

15. Schaefferkoetter JD, Townsend DW. Quantitative accuracy and lesion detectability of low-dose FDG-PET for lung cancer screening. J Nucl Med. 2016;58:399-405.

16. Lodge MA, Chaudry MA, Wahl RL. Noise considerations for PET quantification using maximum and peak standardized uptake value. J Nucl Med. 2012;53:10411047.

17. Akamatsu G, Ikari Y, Nishida $H$. Influence of statistical fluctuation on reproducibility and accuracy of $\mathrm{SUV}_{\max }$ and $\mathrm{SUV}_{\text {peak }}$ : a phantom study. J Nucl Med Technol. 2015;43:222-226.

18. Schmidtlein CR, Beattie BJ, Bailey DL, et al. Using an external gating signal to estimate noise in PET with an emphasis on tracer avid tumors. Phys Med Biol. 2010;55:6299-6326.

19. Armstrong IS, Kelly MD, Williams HA, Mattheus JC. Impact of point spread function modelling and time-of-flight on FDG uptake measurements in lung lesions using alternative filtering strategies. EJNMMI Phys. 2014;1:99.

20. Boellaard R, Delgado-Bolton R, Oyen WJ, et al. FDG PET/CT: EANM procedure guidelines for tumour imaging: version 2.0. Eur J Nucl Med Mol Imaging. 2015;42:328-354.

21. Koopeman D, van Osch JA, Jager PL, et al. Technical note: how to determine the FDG activity for tumor PET imaging that satisfies European guidelines. EJNMMI Phys. 2016;3:22.

22. Akamatsu G, Ishikawa K, Mitsumoto K, et al. Improvement in PET/CT image quality with a combination of point-spread function and time-of-flight in relation to reconstruction parameters. J Nucl Med. 2012;53:1716-1722.

23. de Groot EH, Post N, Boellaard R, Wagenaar NRL, Willemsen ATM, Dalen JA. Optimized dose regimen for whole-body FDG-PET imaging. EJNMMI Res. 2013;3:63.

24. Menezes VO, Machado MAD, Queiroz CC, et al. Optimization of oncological ${ }^{18}$ F-FDG PET/CT imaging based on a multiparameter analysis. Med Phys. 2016; 43:930-938.

25. Wickham F, McMeekin H, Burniston M, et al. Patient-specific optimisation of administered activity and acquisition times for ${ }^{18} \mathrm{~F}$-FDG PET imaging. EJNMMI Res. 2017;7:3.
26. Spadafora M, Pace L, Mansi L. Segmental ${ }^{18}$ F-FDG-PET/CT in a single pulmonary nodule: a better cost/effectiveness strategy. Eur J Nucl Med Mol Imaging. 2017;44:1-4.

27. Veronesi G, Bellomi M, Veronesi U, et al. Role of positron emission tomography scanning in the management of lung nodules detected at baseline computed tomography screening. Ann Thorac Surg. 2007;84:959-965.

28. Lasnon C, Desmonts C, Quak E. Harmonizing SUVs in multicentre trials when using different generation PET systems: prospective validation in non-small cell lung cancer patients. Eur J Nucl Med Mol Imaging. 2013;40:985-996.

29. Quak E, Roux P, Hofman M. Harmonizing FDG PET quantification while maintaining optimal lesion detection: prospective multicentre validation in 517 oncology patients. Eur J Nucl Med Mol Imaging. 2015;42:2072-2082.

30. Lasnon C, Salomon T, Desmonts C, et al. Generating harmonized SUV within the EANM EARL accreditation program: software approach versus EARL-compliant reconstruction. Ann Nucl Med. 2017;31:125-134.

31. EARL procedure for assessing PET/CT system specific patient FDG activity preparations for quantitative FDG PET/CT studies. EANM Research Ltd. website. http://earl.eanm.org/html/img/pool/EARL-procedure-for-optimizing-FDGactivity-for-quantitative-FDG-PET-studies_version_1_1.pdf. Updated January 30, 2017. Accessed December 6, 2018.

32. Wahl RL, Jacene H, Kasamon Y, Lodge MA. From RECIST to PERCIST: evolving considerations for PET response criteria in solid tumors. $\mathrm{J} \mathrm{Nucl} \mathrm{Med}$. 2009;50(suppl 1):122S-150S.

33. Ptaček J, Karhan P, Fiala P. Optimal reconstruction matrix and PET image filtration for point-spread function and time-of-flight reconstruction: a phantom study. Phys Med. 2017;39:95-99.

34. Lodge MA. Repeatability of standardized uptake value in oncologic ${ }^{18}$ F-FDG PET. J Nucl Med. 2017;58:523-532.

35. Boellaard R. Standards for PET image acquisition and quantitative data analysis. J Nucl Med. 2009;50(suppl 1):11S-20S.

36. Aide N, Lasnon C, Veit-Haibach P, Sera T, Sattler B, Boellaard R. EANM/EARL harmonization strategies in PET quantification: from daily practice to multicentre oncological studies. Eur J Nucl Med Mol Imaging. 2017;44(suppl 1):17-31.

37. Adams MC, Turkington TG, Wilson JM, Wong TZ. A systematic review of the factors affecting accuracy of SUV measurements. AJR. 2010;195:310-320.

38. Munk OL, Tolbod LP, Hansen SB, Bogsrud TV. Point-spread function reconstructed PET images of sub-centimeter lesions are not quantitative. EJNMMI Phys. 2017;4:5. 